\title{
A CMOS Voltage-to-Frequency Linearizing Preprocessor for Parallel Plate RF MEMS Varactors
}

\author{
Michael S. McCorquodale, Mei Kim Ding, and Richard B. Brown \\ Department of Electrical Engineering and Computer Science, Solid State Electronics Laboratory, \\ University of Michigan, Ann Arbor, Michigan 48109-2122
}

\begin{abstract}
A generalized parallel plate radio frequency microelectromechanical systems (MEMS) varactor is presented and studied. Its application in an RF oscillator is described and the nonlinear relationship between tuning voltage and realized output frequency is derived. A low power CMOS integrated circuit is proposed to linearize the response. The circuit consumes at most $100 \mu \mathrm{W}$ of power while linearizing the voltage-to-frequency response to a correlation coefficient $\left(R^{2}\right)$ of 0.9988 . The described circuit and theory can be employed in any application where the nonlinear response associated with parallel plate RF MEMS varactors must be compensated.
\end{abstract}

\section{INTRODUCTION}

MEMS technology has been demonstrated successfully in a variety of RF applications including switching [1], filtering, and frequency synthesis [2]. Components such as coupled MEMS varactors [3] and inductors [4] have demonstrated higher quality factor ( $Q$-factor) references for voltage controlled oscillators [3] than alternative integrated technologies. Several parallel plate varactor topologies have been reported with impressive results [3][5]. However, a significant drawback associated with the parallel plate topology is the highly nonlinear tuning response as a function of the electrostatic actuation of the device. Here we discuss the application of a parallel plate MEMS varactor in a typical CMOS $L C$ oscillator. We further examine the mechanical physics of the device and derive the frequency tuning characteristic as a function of the tuning voltage. A linearizing technique is described and a complete circuit for preprocessing the tuning voltage is demonstrated.

\section{RF MEMS PARALLEL PlATE VARACTORS}

\section{A. Topology and Operation}

A generalized parallel plate MEMS varactor is presented in Fig. 1. The device is constructed by mechanically suspending a metal top plate in air above a fixed metal bottom plate. A mechanical suspension network provides support for the top plate as shown. The device presents a nominal capacitance set by the device geometry and the nominal

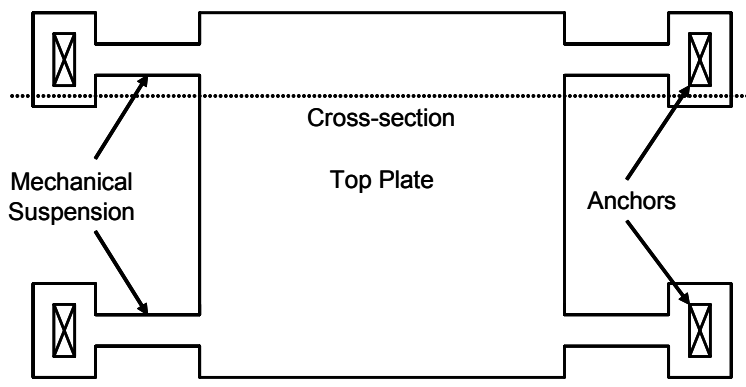

(a)

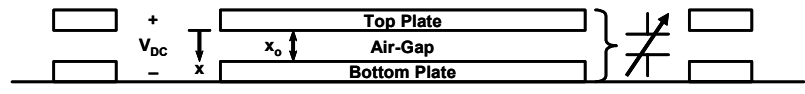

(b)

Fig. 1. A generalized parallel plate RF MEMS varactor (a) Top view illustrating mechanical suspension (b) Cross-section illustrating device operation by electrostatic actuation

gap between the plates, $x_{o}$. By applying a positive DC voltage, $V_{D C}$, across the plates, the moveable top plate will deflect some distance, $x$, due to electrostatic force, thus modulating the capacitance.

\section{B. Application}

A typical application for an RF MEMS varactor is as the tunable element in voltage-controlled-oscillators (VCO). The varactor, when coupled with an inductor, forms an $L C$ tank that provides a high $Q$-factor reference for frequency synthesis that is often superior to alternative integrated technologies, such as those shown in [6]. A typical VCO implementation would include the MEMS varactor coupled with an inductor in a feedback loop sustained by a negative resistance amplifier.

\section{VARACTOR TUNING RESPONSE}

\section{A. Theory}

The fundamental resonant radian frequency, $\omega_{0}$, for a generalized $L C$ oscillator is $\omega_{o}=1 / \sqrt{L C}$, where $L$ is the inductance and $C$ is the capacitance of the tank. When a 
MEMS varactor is employed to realize the capacitance, this variable capacitance is described by the following relationship,

$$
C=\frac{\varepsilon A}{x_{o}-x}
$$

where $\varepsilon$ is the permittivity of air, $A$ is the plate overlap area, $x_{o}$ is the nominal distance between the plates, and $x$ is some displacement forced by the DC tuning voltage, $V_{D C}$ as shown in Fig. 1. The relationship between the varactor capacitance and frequency is found by substitution of (1) into the expression for the fundamental radian frequency.

$$
\omega_{o}=\sqrt{\frac{\left(x_{o}-x\right)}{L \varepsilon A}}
$$

The relationship between $x$ and $\omega$ is clearly nonlinear. However, for the MEMS varactor, $x$ is small compared to $x_{o}$ and at most $x_{o} / 3$ due to device physics. By considering the binomial series of the function $f(x)=\left(x_{o}-x\right)^{1 / 2}$, which is of the same form as (2), one can show that for $x$ much less than $x_{o}$, the relationship is well approximated by the linear term. The correlation coefficient $\left(\mathrm{R}^{2}\right)$ of the least squares linear fit to the displacement-frequency response is 0.9999 for $x$ from 0 to $x_{o} / 3$. Thus, in order to determine the origin of the nonlinear voltage-frequency response, device physics must be investigated.

The electrostatic force, $F_{e}$, generated between the plates by the applied tuning voltage, $V_{D C}$, can be derived by considering the energy, $E$, stored between the plates.

$$
F_{e}=\frac{\partial E}{\partial x}=\frac{1}{2} \frac{\partial C}{\partial x} V_{D C}^{2}=\frac{1}{2} \frac{C V_{D C}^{2}}{\left(x_{o}-x\right)}
$$

The effective electrical spring constant, $k_{e}$, is given by the magnitude of the differential of this force with respect to displacement.

$$
k_{e}=\left|\frac{\partial F_{e}}{\partial x}\right|=\frac{C V_{D C}^{2}}{\left(x_{o}-x\right)^{2}}=\frac{\varepsilon A V_{D C}^{2}}{\left(x_{o}-x\right)^{3}}
$$

A mechanical spring constant, $k_{m}$, is associated with the top plate suspension. A mechanical restoring force, $F_{m}$, is created by this suspension. The relationship between $k_{m}$ and $F_{m}$ is given by Hooke's Law:

$$
F_{m}=k_{m} x
$$

The magnitudes of $F_{m}$ and $F_{e}$ are the same at equilibrium as the electrostatic force is balanced by the mechanical restoring force of the suspension network.

$$
k_{m} x=\frac{1}{2} \frac{C V_{D C}^{2}}{\left(x_{o}-x\right)}
$$

Finally the relationship between $V_{D C}$ and $x$ can be expressed using (6)

$$
V_{D C}=\sqrt{\frac{2 k_{m} x\left(x_{o}-x\right)^{2}}{\varepsilon A}}
$$

Clearly, the origin of the highly nonlinear voltage-frequency response can be attributed to the voltage-displacement response given in (7). Linearization of this response is thus the focus of this work.

\section{B. Linearization Approach and Results}

Three key metrics were considered while deriving an approach in order to linearize the voltage-displacement characteristic. First, the circuit must perform sufficiently accurate linearization of the response. Second, the function realized by the circuit must be reasonably simple to implement with CMOS electronics. Last, the circuit must consume a minimal amount of power.

The response of $V_{D C}$ can be linearized in $x$ exactly by applying the same function in $x$ to $V_{D C}$. However, this function is quite difficult to realize with CMOS electronics. Thus, one might estimate a preprocessed voltage, $V_{p}$, by noting that for small $x$ relative to $x_{o}, V_{D C}$ can be approximated by the following:

$$
V_{D C} \approx \sqrt{\frac{2 k_{m} x x_{o}^{2}}{\varepsilon A}}
$$

Hence, if the applied tuning voltage is preprocessed by applying a square root function (i.e. $V_{p}=V_{D C}{ }^{1 / 2}$ ), the relationship between the tuning voltage and $x$ would be then be nearly linear for small $x$. This approach yields a theoretical voltage-frequency response with a correlation coefficient $\left(R^{2}\right)$ of 0.9870 between the achieved response and the linear function. These results are illustrated in Fig. 2. More accurate linearization can be achieved by expanding (7) to show the relationship between $V_{D C}$ and $x$ is,

$$
V_{D C}=\sqrt{\frac{2 k_{m}}{\varepsilon A}}\left(\sqrt{x} x_{o}-x^{\frac{3}{2}}\right)=a \sqrt{x}-b x^{\frac{3}{2}}
$$

where $a$ and $b$ are constants. Although a square-root function is relatively simple to implement with CMOS electronics, it is quite difficult to realize a $3 / 2$ exponent function. Thus, it was determined empirically that (9) can be well approximated by the following,

$$
a \sqrt{x}-b x^{\frac{3}{2}} \approx c \sqrt{x}+d \ln \left(\frac{x}{e}\right)
$$

where $a, b, c, d$, and $e$ are constants selected such that the fit is accurate. The preprocessed voltage is then of the following form. 


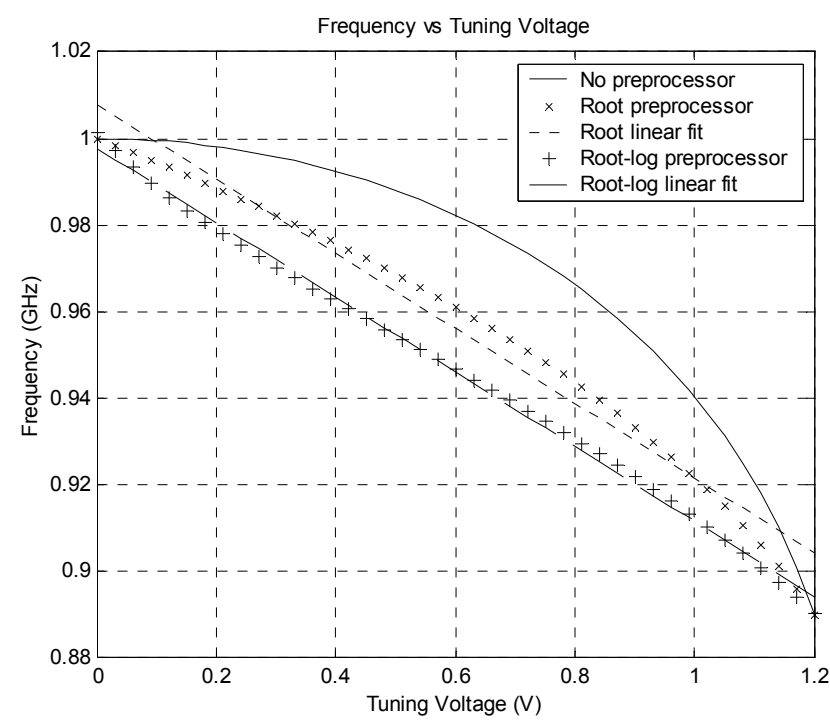

Fig. 2. The applied tuning voltage vs. frequency response with no preprocessing and the two preprocessing approaches derived. The least squares linear fit is shown for both techniques.

$$
V_{p}=c \sqrt{V_{D C}}+d \ln \left(\frac{V_{D C}}{e}\right)
$$

This linearization approach resulted in a theoretical frequency-voltage response with a correlation coefficient $\left(\mathrm{R}^{2}\right)$ of 0.9988 compared to the linear function. The results from both of these approaches are illustrated in Fig. 2. In this work, the latter and more accurate approach is employed.

\section{LINEARIZING CIRCUIT}

\section{A. Topology}

The linearizing circuit ultimately realizes a voltage equal to the sum of the square root of the input voltage and its natural log. This is accomplished by first generating a cur-

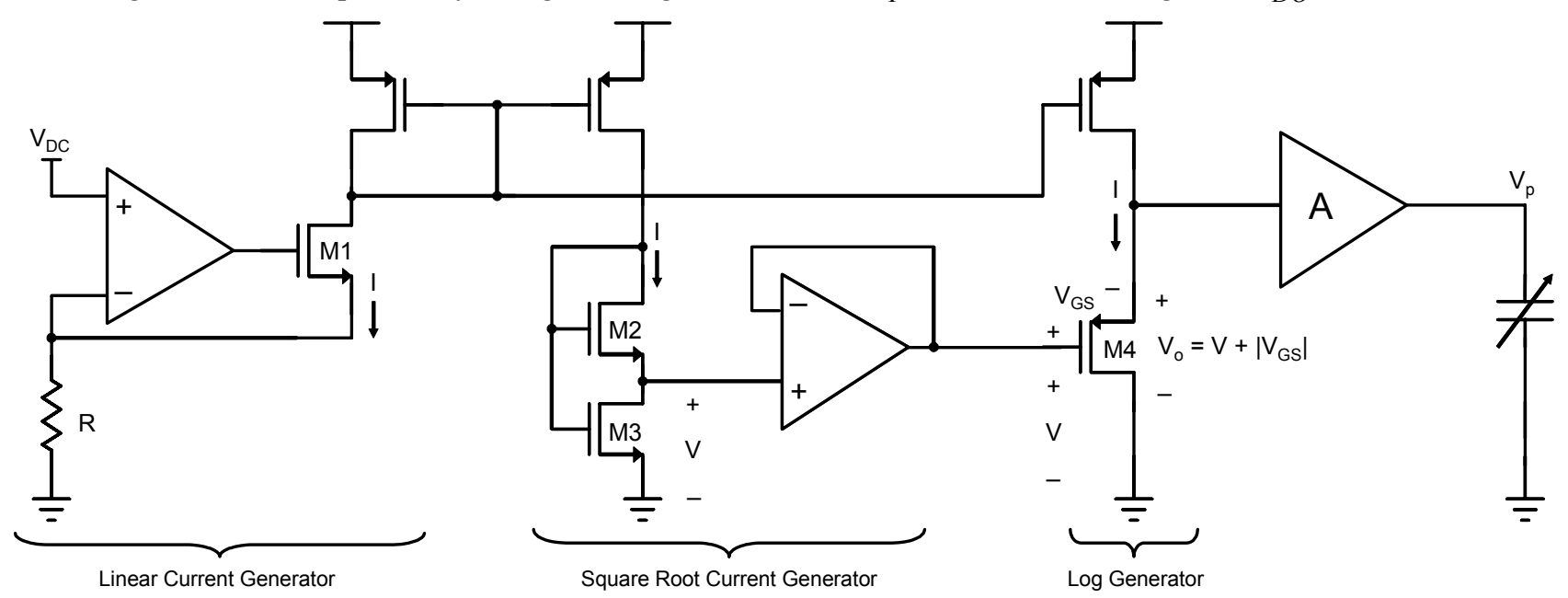

rent that is a linear function of the applied voltage. A simple op-amp voltage-to-current converter [7] is employed for this function as shown in Fig. 3. The transfer function is $I=V_{D C} / R$. The headroom for this circuit is limited by the minimum voltage required to keep M1 on. It is also limited by the magnitude of the current generated as the output of the op-amp will be limited to $V_{D D}$ if the $V_{G S}$ required to maintain the current in M1 is too large. In this application, a small current is generated in order to minimize power consumption and thus the circuit is limited by $V_{G S}$ of $\mathrm{M} 1$. The maximum input voltage is $V_{\max }=V_{D D}-V_{t h}$, where $V_{t h}$ is the threshold voltage of M1.

The nested pair shown in Fig. 3 realizes a square root of current to voltage transformation. As shown in [8], M2 will be forced into saturation while M3 is forced into the linear region of operation. It can be shown that if M2 and M3 are matched, the transfer function is given by,

$$
V=(2-\sqrt{2}) \sqrt{\frac{I}{k}}=(2-\sqrt{2}) \sqrt{\frac{V_{D C}}{R} \frac{1}{k}}
$$

where $k=\mu C_{o x}(W / L)$ and $W / L$ is the device aspect ratio, $\mu$ is the mobility, and $C_{o x}$ is the gate oxide capacitance. Device geometries are chosen to realize the appropriate voltage. A small offset exists when body effect is considered.

The signal is then level shifted through a PMOS device (M4) that is forced into weak inversion, as shown in Fig. 3. The current in this device is also controlled by the linear voltage-to-current generator. In weak inversion and forward saturation the drain current, $I$, of the device is given by [9],

$$
I \approx I_{D O} e^{V_{G S} / n V_{T}}
$$

where $V_{G S}$ is the gate to source voltage, $n$ is the slope factor, $V_{T}$ is the thermal voltage, and $I_{D O}$ is the characteristic

Fig. 3.CMOS root-log voltage-to-frequency linearizing preprocessor for parallel plate RF MEMS varactors 
current given by,

$$
I_{D O}=I_{S} e^{-V_{t h} / n V_{T}}
$$

where $V_{t h}$ is the threshold voltage, and $I_{S}=2 n \mu C_{o x} W / L V_{T}$. Then (13) can be solved to show that:

$$
V_{G S}=n V_{T} \ln \left(I / I_{D O}\right)=n V_{T} \ln \left(V_{D C} / R I_{D O}\right)
$$

Finally the signal is amplified by the DC transfer of a low bandwidth feedback amplifier with gain $A$ as shown in Fig. 3. The amplifier output is high impedance in order to avoid a low impedance AC path to ground from the top plate of the varactor. The realized preprocessed voltage, $V_{p}$, is thus,

$$
V_{p}=A\left((2-\sqrt{2}) \sqrt{\frac{V_{D C}}{R} \frac{1}{k}}+n V_{T} \ln \left(V_{D C} / R I_{D O}\right)\right)
$$

Device geometries and parameters are selected based upon the application and the dynamic range of the tuning voltage.

\section{B. Results}

The described circuit was designed in the $0.18 \mu \mathrm{m}$ mixed-mode process available from Taiwan Semiconductor Manufacturing Company (TSMC). A summary of the relevant parameters is given in Table I. The circuit was designed for a previously developed MEMS varactor with a tuning range of 0 to $1.2 \mathrm{~V}$, which is dictated by the geometry of the mechanical suspension network. The simulated frequency response for the circuit is shown in Fig. 4. According to simulations, the design achieves a correlation coefficient $\left(\mathrm{R}^{2}\right)$ of 0.9988 compared to the linear function, and matches the theoretical performance.

TABLE I

LINEARIZING CIRCUIT PARAMETERS

\begin{tabular}{c|c}
\hline Parameter & Value \\
\hline Process technology & TSMC $0.18 \mu \mathrm{m}$ \\
\hline Devices (including bias) & 25 \\
\hline Supply voltage & $2 \mathrm{~V}$ \\
\hline Power min/max & $7 \mu \mathrm{W} / 100 \mu \mathrm{W}$ \\
\hline Input min/max & $0 \mathrm{~V} / 1.2 \mathrm{~V}$ \\
\hline Output min/max & $0 \mathrm{~V} / 1.2 \mathrm{~V}$ \\
\hline Correlation coefficient $\left(\mathrm{R}^{2}\right)$ & 0.9988 \\
\hline
\end{tabular}

\section{CONCLUSION}

A study of the electromechanical behavior of an RF MEMS varactor was presented. Its application in a VCO was described and the highly nonlinear voltage-frequency relationship was derived. This nonlinear response is related primarily to the relationship between the tuning voltage

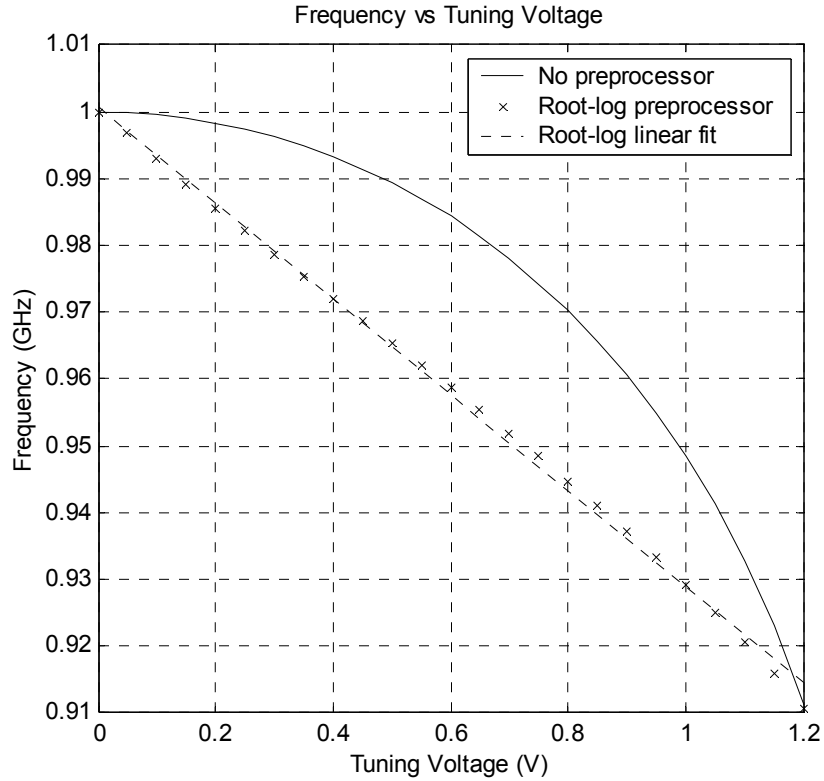

Fig. 4. Frequency response vs. input voltage to preprocessor as compared to the response with no preprocessor.

and the top plate displacement of the varactor. Two linearizing models were presented and the latter, more accurate, was utilized in the design of a CMOS preprocessor using $T S M C$ 's $0.18 \mu \mathrm{m}$ mixed-mode process. The final preprocessing circuit linearizes the voltage-frequency response with a correlation coefficient $\left(\mathrm{R}^{2}\right)$ of 0.9988 .

\section{REFERENCES}

[1] G. M. Rebeiz and J. B. Muldavin, "RF MEMS Switches and Switch Circuits," IEEE Microwave Magazine, vol. 2, issue 4, pp. 59-71, Dec. 2001

[2] C. T.-C. Nguyen, "High-Q micromechanical oscillators and filters for communications (invited)," IEEE ISCAS, Hong Kong, June 9-12, 1997, pp. 2825-2828.

[3] D. Young and B. Boser, "A Micromachined-Based RF LowNoise Voltage-Controlled Oscillator," IEEE CICC, pp. 431434, 1997.

[4] D. Young, et al., "Monolithic High-Performance ThreeDimensional Coil Inductors for Wireless Communication Applications," IEDM, pp. 3.5.1-3.5.4, 1997.

[5] J. Zou, et al., "Development of a Wide Tuning Range MEMS Tunable Capacitor for Wireless Communication Systems," IEDM, pp. 403-406, 2000.

[6] H. Ainspan and J.-O. Plouchart, "A Comparison of MOS Varactors in Fully-Integrated CMOS LC VCO's at 5 and 7 GHz," European Solid-State Circuits Conference, 2000.

[7] R. Gregorian and G. C. Temes, Analog MOS Integrated Circuits for Signal Processing, New York: John Wiley \& Sons, 1986.

[8] I. M. Filanovsky and H. P. Baltes, "Simple CMOS Analog Square-Rooting and Squaring Circuits," IEEE Trans. on Circuits and Systems I, vol. 39, no. 4, April 1992.

[9] E. Vittoz and J. Fellrath, "CMOS Analog Integrated Circuits Based on Weak Inversion Operation," IEEE J. of Solid-State Circuits, vol. SC-12, no. 3, pp. 224-231, June 1977. 